\title{
Analytical Study of Substrate Parasitic Effects in Common-Base and Common-Emitter SiGe HBT Amplifiers
}

\author{
Neti V L Narasimha Murty ${ }^{*}$ and M. Hemalata Rao \\ School of Electrical Sciences, Indian Institute of Technology Bhubaneswar, Bhubaneswar-751013, INDIA \\ murtyn@iitbbs.ac.in*and hlm10@iitbbs.ac.in
}

\begin{abstract}
An analytical study to quantify the substrate parasitic effects on SiGe HBT amplifiers in both common-base and commonemitter configuration is presented. The power gain relations and stability factors are derived from the modelled S-parameters which are computed at a fixed bias point from the small-signal hybrid- $\Pi$ model of SiGe HBT in both configurations. It has been shown that the power gains of SiGe HBT amplifiers in both configurations are degraded when extrinsic and substrate parasitics are taken into account. The degradation in power gains is found to be more pronounced for CB configuration, which makes the design of HBT amplifiers, particularly in the CB mode, difficult. Close matching of the modelled data with the reported experimental results validates the proposed methodology.
\end{abstract}

Index Terms - Common-base, Common-emitter, Maximum Available Gain (MAG), Maximum Stable Gain (MSG), Scattering parameters, SiGe HBT, Stability factor, Substrate parasitics

\section{INTRODUCTION}

Recent technological developments in the area of SiGe heterojunction bipolar transistors (HBTs) have pushed the maximum oscillating frequency $\left(f_{\max }\right)$ and the current gain transition frequency $\left(f_{t}\right)$ values into the millimeter wave range, which were unattainable with the conventional Si devices [1]. $f_{\max }$ values close to $500 \mathrm{GHz}$ have been reported in literature [1]-[2]. Both single and multi-finger emitter configurations with high base doping coupled with the graded Ge base concentration yield high operating frequencies of SiGe HBTs [1]. It is known that many Monolithic Microwave Integrated Circuit (MMIC) foundries characterize the active and passive components based on s-parameter measurements. In addition to the s-parameter data, Mason's unilateral power gain (U) gives information about the maximum oscillating frequency $\left(f_{\max }\right)$ of transistors, but the extracted $f_{\max }$ from U doesn't differ for the same transistor in different configurations, namely common base (CB) and common emitter (CE). Hence the power gain, $G_{\max }$ (Maximum Available Gain/Maximum Stable gain) of transitor is widely used as a important figure of merit while comparing transistor performance in $\mathrm{CE}$ and $\mathrm{CB}$ configuration. The difference in power gain characteristics of SiGe HBT between the CE 
and CB configuration was studied by Ma et al. [3]. Their analytical model suggests that SiGe HBT operated in $\mathrm{CB}$ configuration has higher $\mathrm{G}_{\max }$ than the $\mathrm{CE}$ configuration at higher operating frequencies. They have attributed the superior gain performance of HBT in CB mode to the smaller base resistance. Moreover, in SiGe technology the devices in an IC are isolated using deep trenches on low resistive Si substrates. Eventhough, high resistive substrates are preferable for high frequency applications, due to their complicated fabrication process (e.g. Float Zone-process vs standard Czochralski Si growth), high-resistivity silicon wafers are quite expensive for commercial IC applications [4]. Isolation in circuits realized on silicon substrates in fact is an outstanding topic because a proper isolation technique is a key issue for complex circuits at high frequencies. Some methods were proposed to improve the isolation among the devices integrated onto a monolithic chip [5]. It has been reported that even with proper isolation techniques, substrate parasitics in HBT degrade the performance of the electronic circuits mainly in low-power devices with smaller emitter areas [6]-[7]. Since smaller emitter area (few emitter fingers) devices have low parasitic series resitances compared to large area power devices and hence substrate coupling between and output ports is more significant in few emitter finger HBTs. As the frequency of operation increases, the influence of parasitic substrate effects becomes more important and must be taken into account for accurate designing of HBT amplifiers. Several authors have attempted to modify the small-signal equivalent circuit of HBT by including the substrate effects and have proposed direct parameter extraction techniques from a large set of measured S-parameters for SiGe HBT biased in forward active, cut-off and saturation regions [6], [7]-[10]. Huang et al. [7] have experimentally studied the influence of substrate parasitic effects on power gains of SiGe HBT in both $\mathrm{CB}$ and CE configurations. They have also carried out an analytical study using the modified hybrid- $\Pi$ model of HBT with parasitic substarte capacitances. They have shown that the substrate parasitics indeed deteriorate the maximum available gain of HBT, particularly in CB configuration. However, they haven't studied the effect of substrate parasitics on the stability of the HBT amplifiers. Recently, In this paper we have presented a simple and analytical approach to study the effect of substrate parasitics that couples input and output pads on the power gains of SiGe HBT amplifier in both common-base and common-emitter configurations. For the analysis, we have started with the smallsignal hybrid-ח model reported by Lee [8] which is a the modified Gummel-Poon model with parasitic elements $C_{\text {sub }}$ and $R_{\text {sub }}$. The extrinsic series resistances and inductances of the connecting leads to base, emitter and collector terminals have been included in the complete-small signal equivalent circuit of the HBT. Using the parameter conversions, S-parameters of the two-port HBT amplifier in CB and CE mode have been computed for a matched impedance of $50 \Omega$. Later the amplifier has been checked for stability in the frequency range of $1.2-50 \mathrm{GHz}$ at that bias point including the above parasitic effects. The calculated values of the power gains in the frequency ranges of $0.2-10 \mathrm{GHz}$ are in good agreement with the reported experimental results [8] in the literature. 


\section{GAIN RELATIONS OF HBT AMPLIFIERS}

\section{A. Scattering Parameters}

A simplified schematic structure of a SiGe HBT is shown in Fig.1 with the physical origin of the lumped circuit elements. Most of the small-signal equivalent circuit models of active components are derived from the measured Scattering parameters (S-parameters). Broadly they fall in two categories: T-model and Hybrid $\Pi$ - model. Each one of the above topologies has its own edge over the other. All the model parameters of T-model can be directly derived from the device physics. Whereas the hybrid- $\Pi$ model is the modified version of well-known Gummel-Poon model. Teeter et al. [10] has compared both the circuit topologies of SiGe HBTs up to $100 \mathrm{GHz}$. He has shown the direct correspondence between these two models. He also concluded that the most often used hybrid- $\Pi$ model can be used for accurate circuit simulations with the inclusion of the collector current delay.

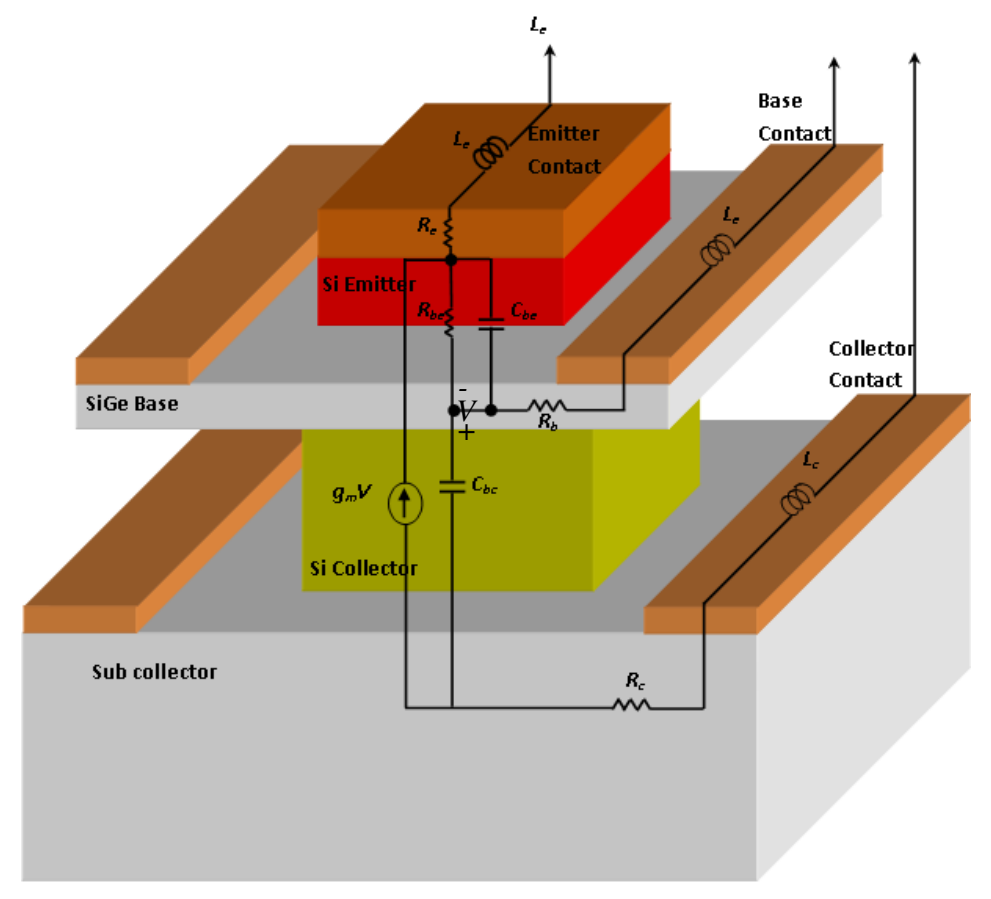

Fig. 1. Schematic structure of a SiGe HBT with the physical origin of lumped circuit intrinsic elements

Several authors have identified the parasitic substrate effects on the performance of SiGe HBT amplifiers at high frequencies [5], [7-9]. They have modified the hybrid $\Pi$-model of HBT to account for the substrate parasitics. In this study we have used the modified small-signal model proposed by Lee [8] to include the substrate parasitic effects. Lee et al. have proposed an improved parameter extraction method for Vertical Bipolar Inter Company (VBIC) -type hybrid П- model of SiGe HBTs by including a non-VBIC-parameter in the small-signal equivalent circuit. Their proposed extraction method successfully characterizes the S-parameters of HBT for broad range of frequencies. 
The small-signal equivalent circuit of the SiGe HBT is shown in Fig.2. Here $R_{b}, R_{e}$ and $R_{c}$ are the extrinsic parasitic resistances of the base, emitter and collector interconnection leads respectively. The intrinsic device model of HBT is shown the inset of Fig. 2 in common emitter (CE) configuration. The base-emitter (B-E) capacitance $\left(C_{b e}\right)$ contains both diffusion and depletion capacitances of the BE junction whereas the base-collector capacitance $\left(C_{b c}\right)$ includes only the depletion capacitance. The transconductance $\left(g_{m}\right)$ contains a time delay component $(\tau)$ which changes the phase of $S_{21}$-parameter at high-frequencies and must be taken into account in calculating the power gains. The time delay $(\tau)$ is actually the base-collector transit time, which is of the order of pico-seconds [8]. The parasitics due to the substrate transistor action is included through $C_{b e p}, C_{s u b}$ and $R_{s u b}$ across the output terminals i.e. collector-emitter [11]. Here $C_{b e p}$ is the parasitic capacitance associated with parasitic transistor action of the base-collector-substrate junctions as defined in VBIC model [8]. Whereas, $C_{\text {sub }}$ and $R_{\text {sub }}$ are related to the substrate parameters of $\mathrm{n}^{+}$- $\mathrm{p}$ collector-substrate junction [6], [8]-[9]. $C_{s c o}$ is a non-VBIC parameter that accounts for small overlapping emitter-collector interconnection metals and its associated capacitance, included by Lee [8].
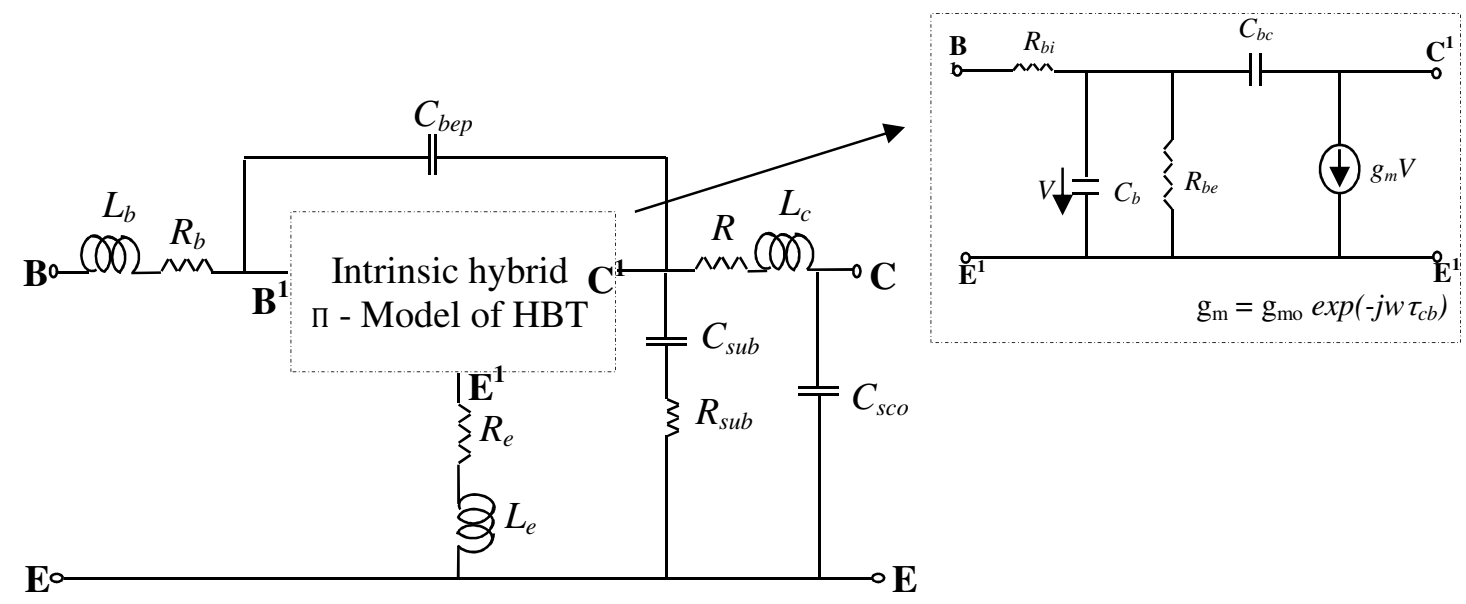

Fig.2. Complete Small-signal model of SiGe HBT in common-emitter configuration. Inset of the figure shows the intrinsic small-signal hybrid- $\Pi$ model of SiGe HBT.

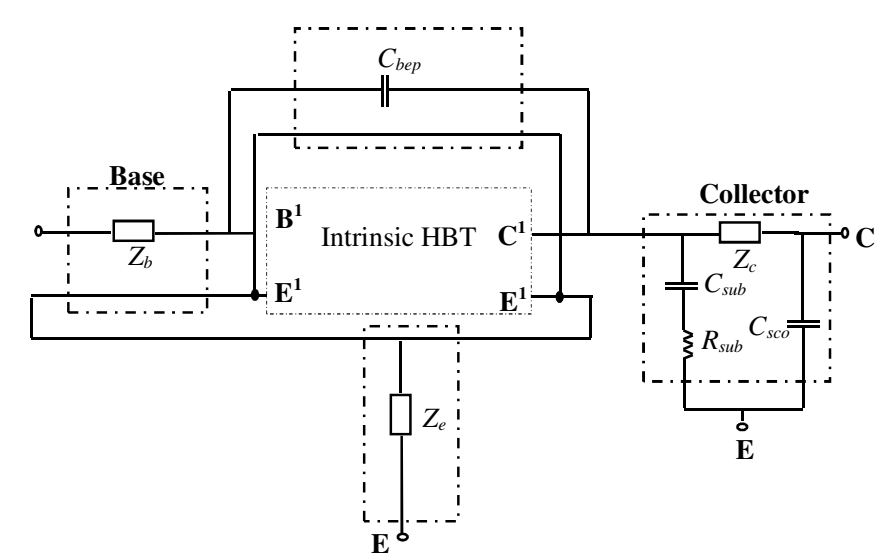

Fig. 3. Complete Small-signal model of SiGe HBT in common-emitter configuration divided into four two-port networks 
The Y-parameters of the intrinsic small signal model of SiGe HBT in CE configuration of Fig. 2 can be expressed as

$$
\begin{gathered}
Y_{11}=\frac{\left(1 / R_{b e}\right)+j \omega C_{b e}+j \omega C_{b c}}{1+R_{b i}\left(1 / R_{b e}+j \omega C_{b e}+j \omega C_{b c}\right)} \\
Y_{12}=\frac{-j \omega C_{b c}}{1+R_{b i}\left(1 / R_{b e}+j \omega C_{b e}+j \omega C_{b c}\right)} \\
Y_{21}=\frac{-g_{m o} e^{-j \omega \tau_{c b}}}{1+R_{b i}\left(1 / R_{b e}+j \omega C_{b e}+j \omega C_{b c}\right)}-j \omega C_{b c} \\
Y_{22}=\frac{g_{m o} e^{-j \omega \tau_{c b} . j \omega C_{b c}}}{\left(1 / R_{b e}+1 / R_{b i}+j \omega C_{b e}+j \omega C_{b c}\right)}+j \omega C_{b c}
\end{gathered}
$$

Whereas the Y-parameters of the intrinsic small-signal model of HBT in CB configuration including substrate parasitics may be given as

$$
\begin{gathered}
Y_{11}=\frac{\left(1 / R_{b e}+j \omega C_{b e}\right)\left(1 / R_{b e}+j \omega C_{b e}+g_{m o} e^{-j \omega \tau_{c b}}\right)}{\left(1 / R_{b e}+1 / R_{b i}+j \omega C_{b e}+j \omega C_{b c}\right)} \\
Y_{12}=\frac{\left(-j \omega C_{b c}\right)\left(1 / R_{b e}+j \omega C_{b e}+g_{m o} e^{-j \omega \tau_{c b}}\right)}{\left(1 / R_{b e}+1 / R_{b i}+j \omega C_{b e}+j \omega C_{b c}\right)} \\
Y_{21}=-g_{m o} e^{-j \omega \tau_{c b}} \frac{\left(1 / R_{b e}+j \omega C_{b c}\right)\left(j \omega C_{b c}+g_{m o} e^{-j \omega \tau_{c b}}\right)}{\left(1 / R_{b e}+1 / R_{b i}+j \omega C_{b e}+j \omega C_{b c}\right)} \\
Y_{22}=j \omega C_{b c}\left[1-\frac{\left(j \omega C_{b c}-g_{m o} e^{-j \omega \tau_{c b}}\right)}{\left(1 / R_{b e}+1 / R_{b i}+j \omega C_{b e}+j \omega C_{b c}\right)}\right]
\end{gathered}
$$

where $g_{m}$ is replaced by $g_{m o} e^{-j \omega \tau_{c b}} . \tau_{c b}$ is the base-collector transit time [8],[10].

Relying on network partitioning and parameter conversion with matched impedance $\left(Z_{o}\right)$ of $50 \Omega$, Sparameters of the complete small-signal model can be computed from the modeled Y-parameters in both configurations [12].

The procedure that we followed for computing S-parameters in CE mode is as follows:

1. The Y-parameters of the intrinsic model, as in eqn (1) are added to the Y-parameters of feedback network $\left(\mathrm{C}_{\text {bep }}\right)$.

$$
\left[\begin{array}{ll}
Y_{11} & Y_{12} \\
Y_{21} & Y_{22}
\end{array}\right]_{\text {Int }+F B}=\left[\begin{array}{ll}
Y_{11} & Y_{12} \\
Y_{21} & Y_{22}
\end{array}\right]_{\text {int }}+\left[\begin{array}{ll}
Y_{11} & Y_{12} \\
Y_{21} & Y_{22}
\end{array}\right]_{F B}
$$

2. The resultant Y-parameters are converted to ABCD-representation and multiplied with the ABCDparameters of base network.

$$
\left[\begin{array}{ll}
A & B \\
C & D
\end{array}\right]_{\text {Int }+F B+b a s e}=\left[\begin{array}{ll}
A & B \\
C & D
\end{array}\right]_{\text {Int }+F B}\left[\begin{array}{cc}
1 & Z_{b} \\
0 & 1
\end{array}\right]_{\text {base }}
$$

3. The resultant ABCD-parameters are converted to Z-parameters and added to the Z-parameters of emitter lead since they are in series.

$$
\left[\begin{array}{ll}
Z_{11} & Z_{12} \\
Z_{21} & Z_{22}
\end{array}\right]_{H B T \text {-collector }}=\left[\begin{array}{ll}
Z_{11} & Z_{12} \\
Z_{21} & Z_{22}
\end{array}\right]_{\text {Int }+F B+\text { base }}+\left[\begin{array}{ll}
Z_{e} & Z_{e} \\
Z_{e} & Z_{e}
\end{array}\right]_{\text {emitter }}
$$

4. The previously obtained Z-parameters are converted to ABCD-parameters and multiplied with the ABCD parameters of collector network. 


$$
\left[\begin{array}{ll}
A & B \\
C & D
\end{array}\right]_{H B T}=\left[\begin{array}{ll}
A & B \\
C & D
\end{array}\right]_{H B T-\text { collector }}\left[\begin{array}{ll}
A & B \\
C & D
\end{array}\right]_{\text {collector }}
$$

5. Finally, the ABCD parameters are converted to S-parameters with $50-\Omega$ matched load [13]. Similarly the S-parameters of HBT in CB mode are computed similar to the above procedure and using the intrinsic Y-parameters of HBT (eqns (2a)-(2d)).

\section{B. Power Gain calculations}

Since the modeled S-parameters of HBT in both configurations are functions of the intrinsic elements, extrinsic components and substrate parasitics through $R_{\text {sub }}$ and $C_{\text {sub }}$, the corresponding power gains are strongly dependent on the above parameters particularly on the substrate parasitic for few-emitter finger HBTs.

To evaluate the performance of SiGe HBT amplifiers, different definitions of power gains can be used which are derived from the modeled S-parameters [12]. Stability of an amplifier is also important if one wants to design it to work in low-frequency, high-gain regime. Stability factor $(\mathrm{K})$ is a parameter that gives information about the stability of the amplifier in the frequencies of interest [14]. $\mathrm{K}$ can be determined from the S-parameters of the HBT as

$$
K=\frac{1-\left|S_{11}\right|^{2}-\left|S_{11}\right|^{2}+\left|S_{11} S_{22}-S_{12} S_{21}\right|}{2\left|S_{12}\right|\left|S_{21}\right|}
$$

If $\mathrm{K}$ is greater than one, the amplifier is unconditionally stable (it will not oscillate for any impedance present at input and output) and in this frequency regime where $\mathrm{K}>1$, the maximum transducer power gain $\left(G_{\max }\right)$ of SiGe HBT amplifier is equal to Maximum Available Gain (MAG) is given by [13]

$$
G_{\max }=M A G=\frac{\left|S_{21}\right|}{\left|S_{12}\right|}\left(K-\sqrt{K^{2}-1}\right)
$$

Whereas the $G_{\max }$ of HBT when $K<1$ is equal to the Maximum Stable Gain (MSG) and is given by [14]

$$
G_{\max }=M S G=\frac{\left|S_{21}\right|}{\left|S_{12}\right|}
$$

Mason's unilateral gain (U) of the SiGe HBT can be calculated from the S-parameters by [14]

$$
U=\frac{\left|\frac{S_{21}}{S_{12}}-1\right|^{2}}{2 . K \cdot\left|\frac{S_{21}}{S_{12}}\right|-2 . \operatorname{Re}\left(\frac{S_{21}}{S_{12}}\right)}
$$

Where $\mathrm{K}$ is the stability factor expressed in eqn (7).

\section{SIMULATION RESULTS AND DISCUSSION}

Since S-parameters of HBT are bias and frequency dependent, we have computed the Sparameters at a fixed bias i.e. for fixed intrinsic and extrinsic parameters for a fixed voltages and currents [8]. For simplicity, we have used the extracted intrinsic and extrinsic parameters by Lee et al. [8] which are $R_{b i}=8.56 \Omega, C_{b e p}=4.26 f \mathrm{~F}, C_{s c o}=15.2 \mathrm{f}, R_{b e}=2405 \Omega, C_{b e}=0.465 p \mathrm{~F}, g_{m o}=81 \mathrm{mS}, \tau_{\mathrm{cb}}=$ 
$1.75 \mathrm{ps}, C_{b c}=33.80 f \mathrm{~F}, C_{\text {sub }}=23.93 \mathrm{fF}, R_{\text {sub }}=1.926 \mathrm{k} \Omega, R_{b}=2.16 \Omega, R_{e}=1.91 \Omega, R_{c}=3.32 \Omega$ and the parasitic lead inductance values are $L_{b}=20 \mathrm{pH}, L_{e}=3.4 \mathrm{pH}$ and $L_{c}=22 \mathrm{pH}$ respectively.

The S-parameters of the SiGe HBT were computed using the lumped small-signal equivalent circuit model of HBT, as in Fig. 2. The computed S-parameters at a fixed bias-point in CE configuration using the above mentioned parameters is shown in Fig. 4 in the frequency range from 0.2-10.2 GHz. The modelled S-parameters qualitatively track the behavior reported in [8] typical to HBT amplifiers. The effect of the substrate parasitics on the S-parameters of the SiGe HBT in CE configuration is simulated and shown in Fig. 5. Note that the scaling factor of $S_{21}$ parameter is much lower than that of $S_{12}$ parameter. As one can see, substrate parasitic effects severly degrade the $S_{21}$ parameter (forward gain) of the HBT at higher frequencies. We have further computed $\left|h_{21}\right|^{2}$ and the power gain $\left(\mathrm{G}_{\max }\right)$ using the modeled equations. Whereas, $\mathrm{h}_{21}$ is computed from the standard parameter conversion from S-parameters to h-parameters [13]. As the frequency of operation increases, Fig. 6 shows reduction in both the magnitude of square of $\mathrm{h}_{21}$ and $\mathrm{G}_{\max }$ which is attributed to the presence of the frequency dependent parasitic elements. To check the valildity, we have compared our modeled results with that of the reported experimental results of [8]. Very good matching with the reported results complements the proposed methodology. Fig.7 compares the intrinsic power gain of HBT amplifier in both CE and CB configuration, without extrinsic parasitic elements in the frequency range from 1.2-50 GHz. The frequency of opertaions in the simulations has been extended to further compare the gain improvements/degradation in one configuration compared to the other. As evident from the results that the intrinsic HBT operated in CB configuration offers improvement in the power gain which is expected because of the low base resistance (due to high base doping). The stability factor (K) of HBT amplifiers without parasitics in both $\mathrm{CE}$ and $\mathrm{CB}$ configurations is also studied and is shown in Fig. 8. The frequency above which the amplifier is stable is around $27 \mathrm{GHz}$ for $\mathrm{CB}$ mode and a lower value of around $15 \mathrm{GHz}$ for $\mathrm{CE}$ mode. It is evident that the intrinsic HBT in common-base mode gives better gain and more stable than the corresponding CE mode of operation.

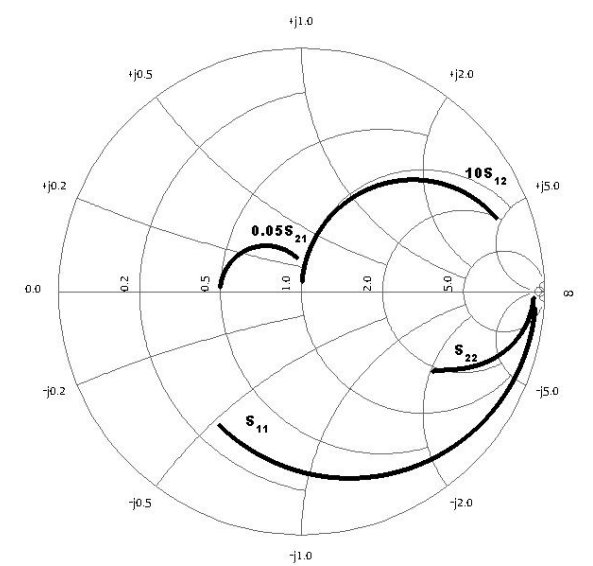

Fig. 4. Simulated S-parameters of SiGe HBT in Common Emitter Configuration in the frequency range of 0.2-10 GHz. 


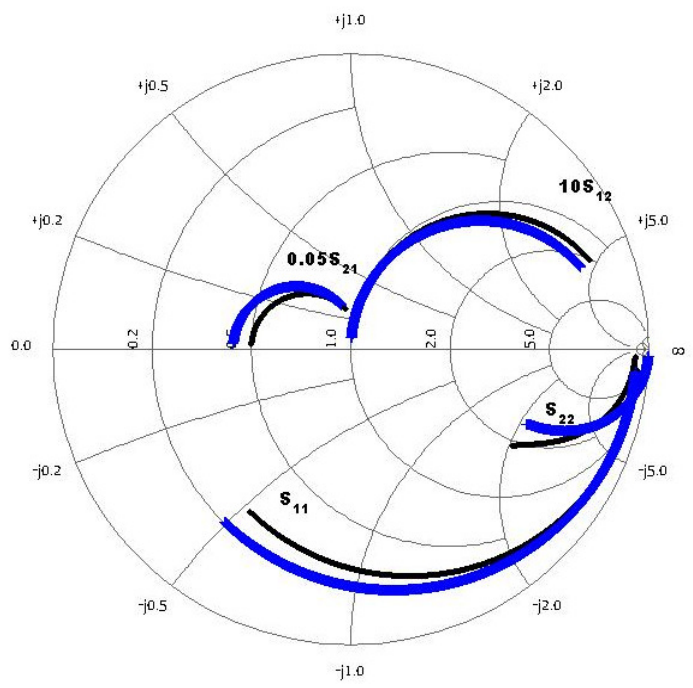

Fig. 5. Simulated S-parameters of SiGe HBT in the frequency range of $0.2-10 \mathrm{GHz}$ with substrate parasitics (black solid line) and without substrate parasitics (blue solid line).

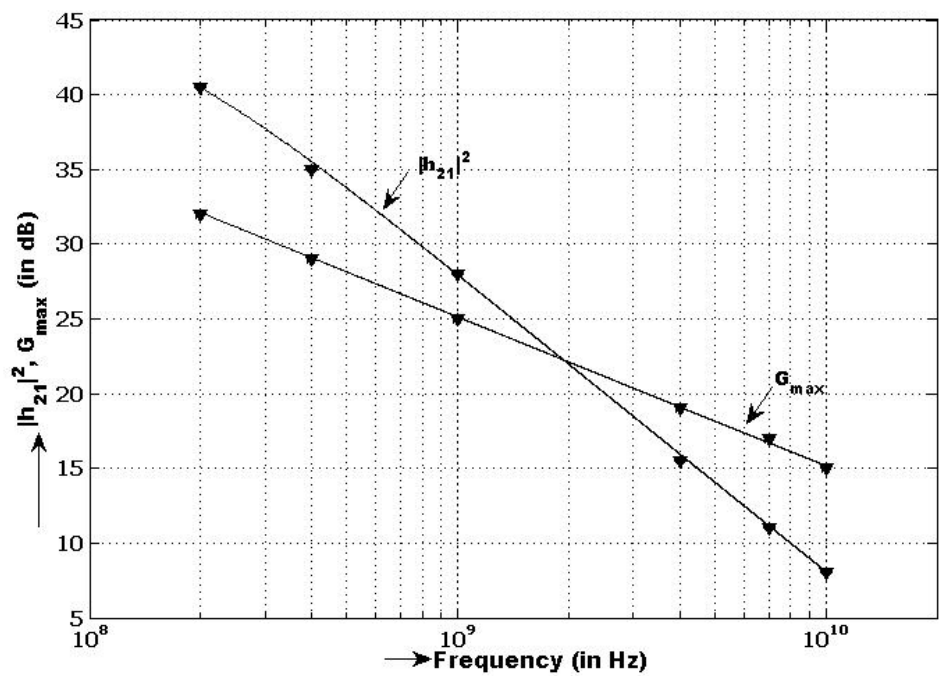

Fig. 6. Calculated (-) and reported ( ) [8] $\downarrow_{21} 1^{2}$ and the power gain $\left(\mathrm{G}_{\mathrm{max}}\right)$ of SiGe HBT in the frequency range of 0.2-10 GHz.

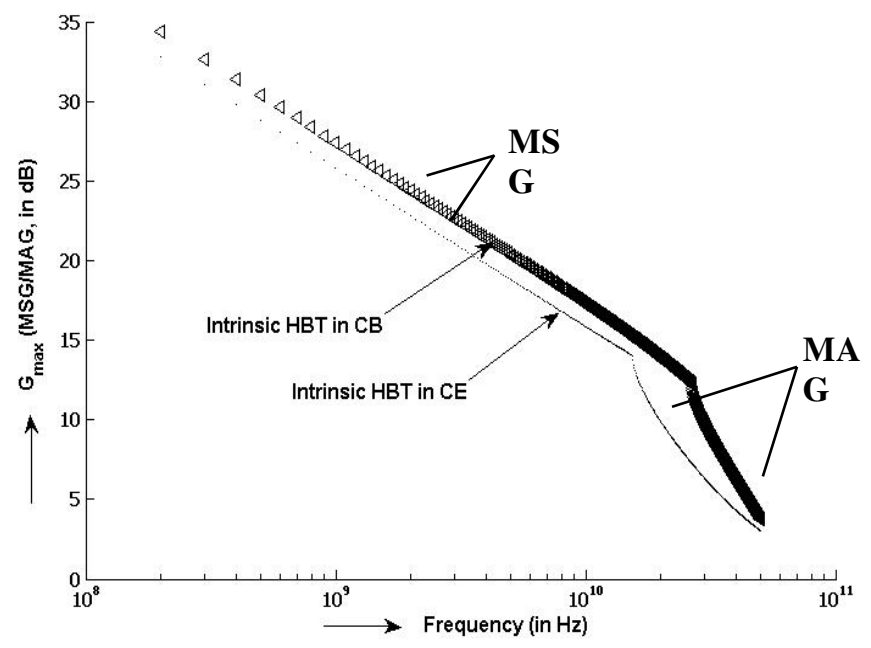

Fig.7. Intrinsic power gain $\left(\mathrm{G}_{\max }\right)$ of a SiGe HBT opertaed in active region in both $\mathrm{CE}$ and $\mathrm{CB}$ configurations calculated in the frequency range $1.2-50 \mathrm{GHz}$. 


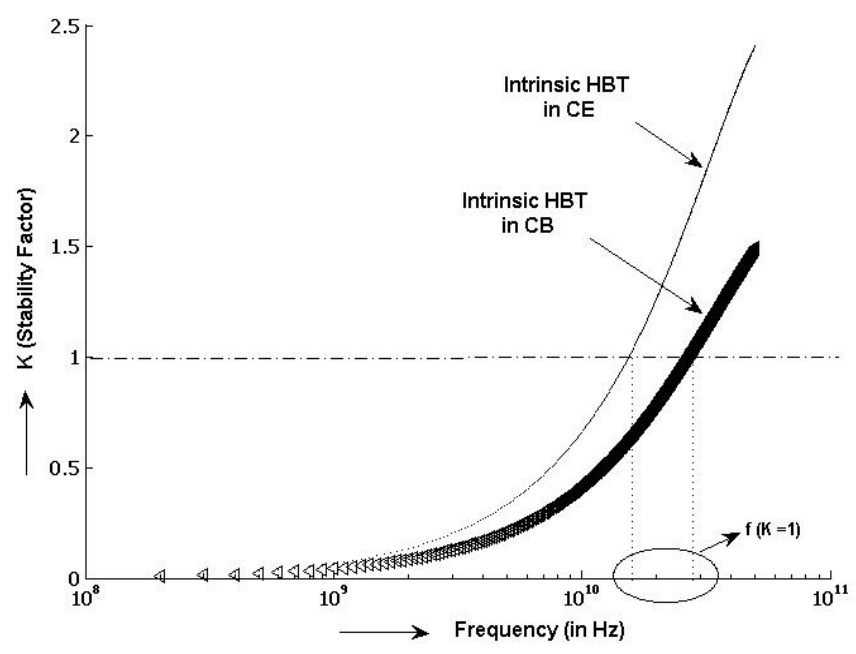

Fig. 8. Stability factor (K) vs Frequency of intrinsic SiGe HBT opertaed in active region in both CE and CB configurations. Note that $f(K=1)$, i.e. frequency at which $K=1$ for $C B$ is $27 \mathrm{GHz}$ and for $\mathrm{CE}$ is $15 \mathrm{GHz}$ for the bias point mentioned.

The advantage of the intrinsic SiGe HBT in CB mode is found to be lost in presence of substrate parasitics as shown in Fig. 9 for typical mid frequencies of interest. It is found that substrate and lead parasitics deteriorate the power gain of $\mathrm{CB}$ amplifier. The substrate parasitics also extends the frequency range where the amplifier is unstable as simulated and shown in Fig. 10. The frequency at which $\mathrm{K}=1$ for $\mathrm{CB}$ mode with parasitics is around $43 \mathrm{GHz}$ for given parameters as opposed to 27 $\mathrm{GHz}$ without substrate parasitics. Hence, it is found that the substrate parasitics not only reduces the gain but also increases the unstable region of the spectrum.

To further study the sensitivity of power gain to the substrate parasitics we have simulated the power gains of HBT in both configurations with and without substrate parasitics and are shown in figures 11 and 12. It is observed that the maximum power gain of HBT in CB mode is dramatically reduced as compared to CE mode. The substrate effects on CB HBT need to be mitigated in order to fully utilize the higher intrinsic power gain in CB mode than in CE mode. Further investigation is required to solve this critical issue.

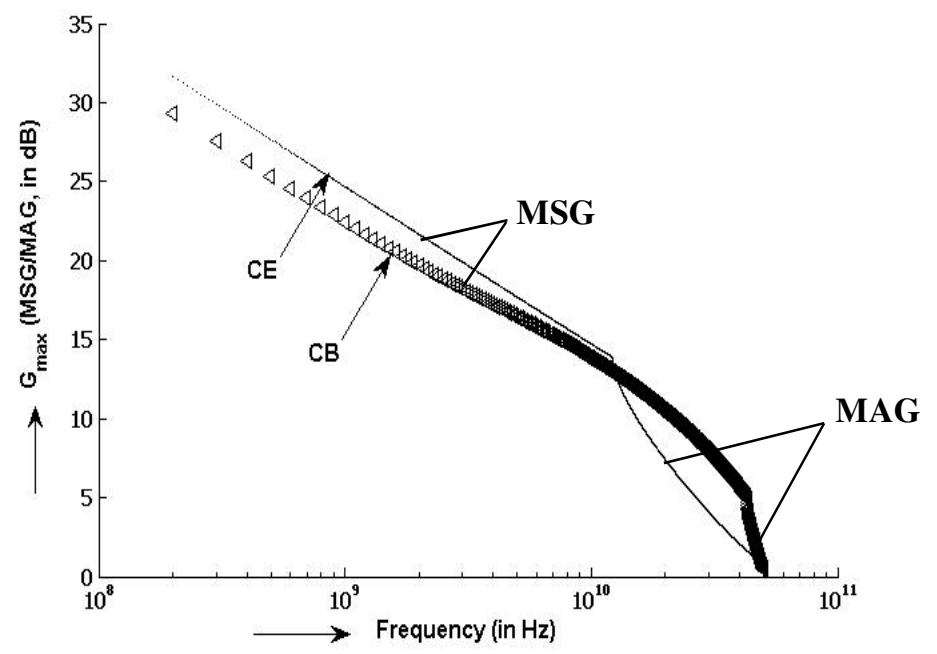

Fig. 9. Simulated power gain $\left(\mathrm{G}_{\max }\right)$ of SiGe HBT in the frequency range of 1.2-50 GHz in both $\mathrm{CB}$ and $\mathrm{CE}$ configurations with all possible parasitic elements. 


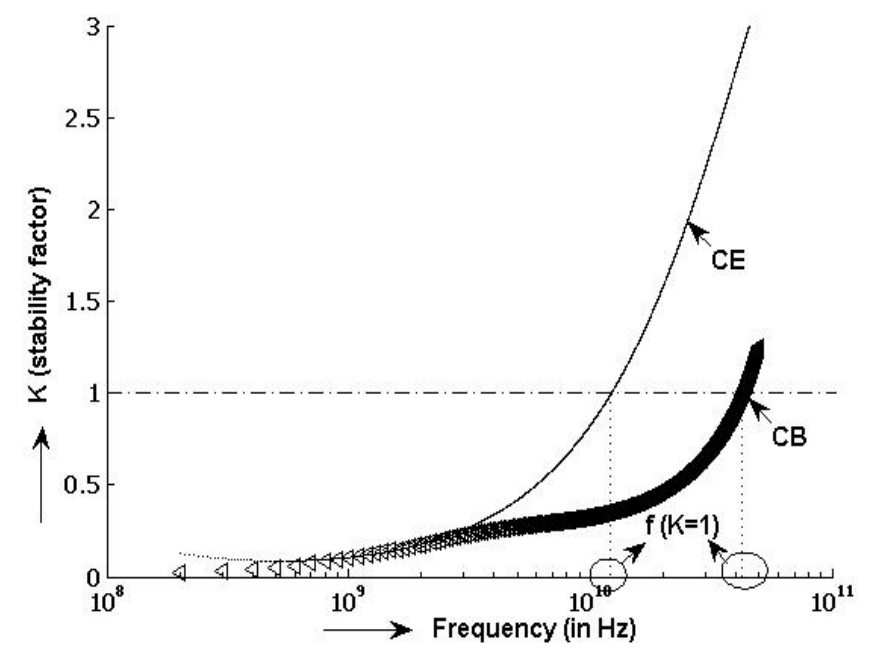

Fig. 10. Stability factor for different frequencies of SiGe HBT in CE and CB configurations with parasitic elements. $\mathrm{f}(\mathrm{K}=1)$ for $\mathrm{CB}$ is around $43 \mathrm{GHz}$ and for $\mathrm{CE}$ is $14 \mathrm{GHz}$ for the bias point mentioned.

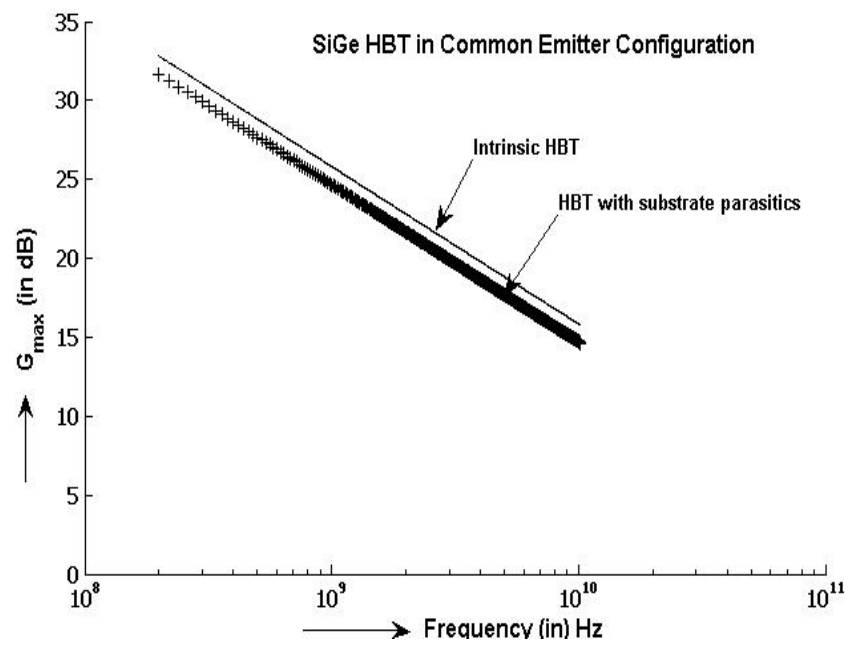

Fig. 11 Simulated $G_{\max }$ vs frequency of HBT in CE mode showing the effect of substrate parasitics in the frequency range 0.2-10.2 GHz.

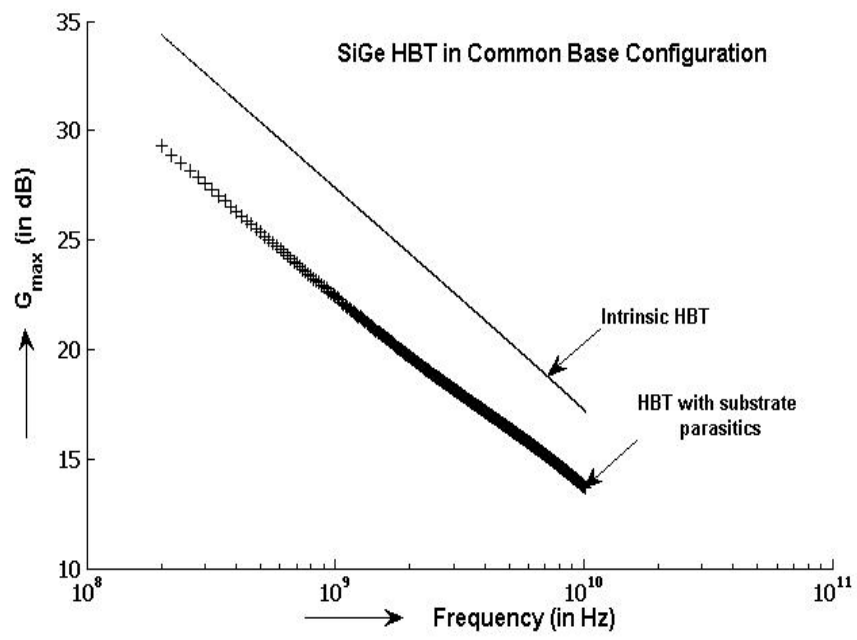

Fig. 12 Variation of Power gain of SiGe HBT for different frequencies in the range $0.2-10.2 \mathrm{GHz}$ with and without substrate parasitics. 
It is well known that the transition frequency $\left(f_{t}\right)$ of a SiGe HBT is an important figure of merit since it determines the operating frequency at which the common-emitter, short circuit current gain $\left(h_{f e}\right)$ decreases to unity. The $f_{t}$ can be extracted by extrapolating $\left|h_{21}\right|^{2}$ Vs $f$ curve (such as in Fig.6) of the HBT. However, the operation of the HBT at higher frequencies above $f_{t}$ is still possible beacuse of the gain available due to mismatch between input and output. The maximum frequency of oscillation $\left(f_{\max }\right)$ is the frequency at which the power gain of a HBT drops to unity. The $f_{\max }$ of SiGe HBT can also be derived based on unilateral gain, using eqn.10, by finding out the frequency at which $\mathrm{U}=1$. From practical standpoint, $\mathrm{U}$ is easier to measure at realtively low frequencies as compared to MAG requires measurements upto higher frequencies. Moreover, the $f_{\max }$ extracted from $\mathrm{U}$ data is always found to be higher than extracted from power gain (MAG) data, and hence is a natural choice being used by many groups [15]. Hence, in order to study the effect of substrate parasitics on the maximum oscillating frequency $\left(f_{\max }\right)$ of the SiGe HBT the variation of the calculated Mason's unilateral power gain (U) with frequency, calculated using eqn. (10), is plotted in Fig. 13. It is observed that the unilateral gain (U) of the SiGe HBT decreases with increae in frequencies in both cases. The value of $f_{\max }$ of the SiGe HBT without parasitics is found to be $70.65 \mathrm{GHz}$ where as the substrate parasitics are found to reduce the $f_{\max }$ from $70.65 \mathrm{GHz}$ to $49 \mathrm{GHz}$.

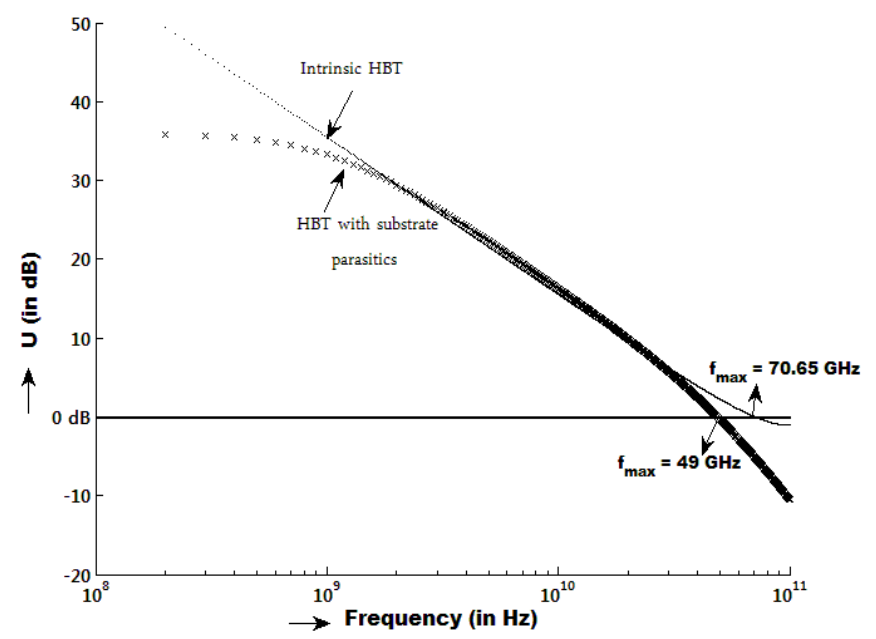

Fig. 13 Variation of Mason's unilateral gain (U) of SiGe HBT for different frequencies in the range 0.2-100 GHz with and without substrate parasitics. The extracted $f_{\max }$ from $\mathrm{U}$ without parasitics is found to be $70.65 \mathrm{GHz}$ and $f_{\max }$ with parasitics is approximately $49 \mathrm{GHz}$.

\section{CONCLUSIONS}

In this paper, an improved and accurate analytical approach to study the effect of substrate parasitics on the power gain relations of SiGe HBT in both $\mathrm{CB}$ and $\mathrm{CE}$ configurations was presented. The methodology was based on modeling the S-parameters derived from the complete small-signal hybrid-ח model of SiGe HBT including intrinsic, extrinsic and substrate parasitic elements. The influence of substrate parasitics are simulated with the help of the modeled S-parameters. The power 
gains (MSG/MAG) of the SiGe HBT were calculated using the modeled S-parameters with and without substrate parasitics analytically. The excellent agreement between modeled and experimental work reported in literature has proven the accuracy of the proposed methodology. It is found that the superior gain characteristics of intrinsic CB SiGe HBT amplifier over CE amplifier are lost in presence of substrate parasitics. Substrate parasitic effects further increase the instability by reducing the stability factor $(\mathrm{K})$ for a given frequency in both configurations. Our simulation results showed that this degradation is more pronounced for HBT operated in CB mode than in CE mode, which makes the HBT design extremely in-efficient in CB mode than corresponding CE mode. Hence proper care must be taken in designing HBT amplifiers particularly in the CB mode. It is also observed that the parasitics in SiGe HBT degrade the maximum frequency of oscillation $\left(f_{\max }\right)$ in addition to the unilateral gain $(\mathrm{U})$. Other ways of minimizing the substrate effects need to be investigated.

\section{REFERENCES}

[1] B. Heinemann, A. Fox and H. Rücker, “Advanced transistor architectures for half-terahertz SiGe HBTs," ECE Trans., vol.50, pp.61-71, 2013.

[2] P. Chevalier, T. F. Meister, B. Heinemann, S. Van Huylenbroeck, W. Liebl, A. Fox, A. Sibaja-Hernandez and A. Chantre, "Towards THz SiGe HBTs," IEEE Bipolar/BiCMOS Circuits and Technology Meeting (BCTM), 2011, pp.5765.

[3] Z. Ma and N Jiang, "On the operation Configuration of SiGe HBTs based on power gain analysis," IEEE Trans. Electron Devices, vol. 52, pp. 248-255, 2005.

[4] J. Lee, Y. Kim, E. Lee, C. Kim and P. Roblin, “A 8-GHz SiGe HBT VCO design on a low resistive silicon substrate using GSML,” IEEE Trans. Circuits. Syst. I, vol.54, pp.2128-2136, 2007.

[5] E. Sönmez, S. Chartier, A. Trasser and H. Schumacher, “ Isolation issues in multifunctional Si/SiGe ICs at 24GHz," in International Microwave Symposium, 2005, pp. 12-17.

[6] T. K. Johansen, J. Vidkjær and V. Krozer, "Substrate effects in SiGe HBT modeling," in GaAs IC Symp. Tech. Dig., 2003, pp.445-448.

[7] H. Huang, Z. Ma, P. Ma and M. Ranennelli, "Influence of substrate parasitic effects on power gain relation between CE and CB SiGe HBTs," in Silicon Monolithic Integrated Circuits in RF systems, 2008, pp. 62-65.

[8] K. Lee, K. Choi, S. Kook, D. Cho, K. park and B. Kim, “ Direct parameter extraction of SiGe HBTs for the VBIC bipolar compact model,” IEEE Trans. Electron Devices, vol.52, pp.375-384, 2005.

[9] B. Han, H. Wang and J. Gao, “ An approach for SiGe HBT small-signal modeling up to $40 \mathrm{GHz}$," Intl. Journal of Electronics, 2013, DOI:10.1080/00207217.2013.769182.

[10] D. A. Teeter and W. R. Curtice, "Comparison of hybrid Pi and Tee HBT circuit topologies and their relationship to large signal modeling," in IEEE-MTT S Digest, 1997, pp.375-378.

[11] U. Basaran, N. Wieser, G. Feiler and M. Berroth, "Small-signal and high frequency noise modeling of SiGe HBTs," IEEE Tran. Microwave Theory and Tech., vol.53, pp.919-928, 2005.

[12] Neti V L Narasimha Murty and S. Jit," Analytical modeling of photo-effects on s-parameters of GaAs MESFETs,", Microwave Optical Technol Lett, vol.48, pp. 150-155, 2006.

[13] S. Marsh, Practical MMIC Design, Boston: Artech House, 2006.

[14] R. Ludwig and G. Bogdanov, RF Circuit Design: Theory and Applications, New Jersey: Pearson Edu, 2009.

[15] J. D. Cressler and G. Niu, Silicon-Germanium Heterojunction Bipolar Transistors, Boston: Artech House, 2003. 